\title{
Assistência de enfermagem às mulheres em situação de violência sexual: revisão integrativa
}

\author{
Nursing care for women in situations of sexual violence: integrative review
}

Asistencia de enfermería a las mujeres en situación de violencia sexual: revisión integradora

\author{
Davydson Gouveia Santos' @ ; Evangelia Kotzias Atherino dos Santos' ${ }^{\circledR}$; Marli Terezinha Stein Backes ${ }^{\circledR}$;

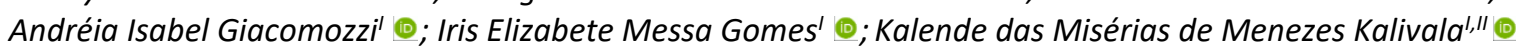

'Universidade Federal de Santa Catarina, Florianópolis, SC, Brasil; "Instituto Superior Politécnico da Caála, Huambo, Angola

\begin{abstract}
RESUMO
Objetivo: descrever a atuação do enfermeiro no atendimento às mulheres em situação de violência sexual a partir da literatura Método: pesquisa bibliográfica na modalidade revisão integrativa da literatura, com busca dos estudos primários publicados entre 2015 a 2019, realizada em abril de 2020, em sete bases de dados, sendo selecionados e analisados dez artigos. Resultados: da síntese das evidências, emergiram três categorias: assistência clínica medicamentosa, assistência clínica não-medicamentosa e falta de qualificação profissional, revelando insatisfação de algumas mulheres no atendimento. Conclusão: os estudos analisados descrevem com clareza a assistência de enfermagem prestada de imediato às mulheres pós agressão sexual, porém, não abordam a continuidade do processo, constituindo-se essa uma importante lacuna. A síntese de conhecimento produzido oferece subsídios valiosos para a melhoria da qualidade da assistência de enfermagem às mulheres em situação de violência e ratifica a importância dos seus resultados para fundamentar a prática clínica nesta área de estudo.

Descritores: Enfermagem; Cuidados de Enfermagem; Violência contra a Mulher; Violência Sexual.
\end{abstract}

\begin{abstract}
Objective: from the literature, to describe the role of nurses in assisting women in situations of sexual violence. Method: bibliographic search in seven databases, in April 2020, for integrative literature reviews, searching for primary studies published between 2015 and 2019, in which ten articles were selected and examined. Results: from the synthesis of the evidence, three categories emerged: clinical drug care, clinical non-drug care and lack of professional qualification, revealing some women's dissatisfaction with the care. Conclusion: the studies examined clearly describe the nursing care provided immediately to women after sexual assault. However, they do not address continuity of the care, which is an important gap. The knowledge synthesized offered valuable input to improving the quality of nursing care for women in situations of violence and confirmed the importance of the findings to supporting clinical practice in this field of study.
\end{abstract}

Descriptors: Nursing; Nursing Care; Violence against Women; Sex Offenses.

\section{RESUMEN}

Objetivo: describir el rol de las enfermeras en la asistencia a las mujeres en situación de violencia sexual a partir de la literatura. Método: investigación bibliográfica en forma de revisión integradora de la literatura, realizada en abril de 2020 , con búsqueda sobre estudios primarios publicados entre 2015 y 2019, en siete bases de datos. Fueron seleccionados y analizados diez artículos. Resultados: de la síntesis de las evidencias emergieron tres categorías: atención clínica con uso de medicación, atención clínica sin uso de medicación y falta de calificación profesional, poniendo en evidencia el descontento de algunas mujeres en cuanto a la atención. Conclusión: los estudios analizados describen claramente los cuidados de enfermería brindados prontamente a las mujeres luego de la agresión sexual, sin embargo, no abordan la continuidad del proceso, lo que constituye un vacío importante. La síntesis de conocimientos producidos ofrece valiosos subsidios para mejorar la calidad de la atención de enfermería a las mujeres en situación de violencia y confirma la importancia de sus resultados para fundamentar la práctica clínica en esta área de estudio.

Descriptores: Enfermería; Atención de Enfermería; Violencia contra la Mujer; Delitos Sexuales.

\section{INTRODUÇÃO}

A Política Nacional de Redução da Morbimortalidade por Acidentes e Violência (PNRMAV) foi lançada em 2001 pelo Ministério da Saúde, oficializando violência como tema a ser trabalhado no setor da saúde no Brasil. Dentre tantos problemas de saúde pública existentes em nosso país, existe o problema da violência, que pela sua magnitude ganha destaque a cada dia, sendo responsável por provocar lesões, traumas físicos e mentais, responsável por diminuir a qualidade de vida das pessoas, podendo até, de forma precoce, levar à morte ${ }^{1}$.

O relatório da Organização Mundial da Saúde (2018) revela que aproximadamente 35\% das mulheres sofreram violência física ou sexual por parceiro íntimo ou não entre 2012 e 2016, mantendo a incidência com relação ao relatório

Agradecimentos ao apoio da Coordenação de Aperfeiçoamento de Nível Superior - Brasil (CAPES) - Código de Financiamento 001; e ao Programa de Pós-Graduação em Psicologia da Universidade Federal de Santa Catarina (PPGP, UFSC) pelo apoio financeiro para tradução.

Autor Correspondente: Davydson Gouveia Santos. E-mail: davydson_gs@hotmail.com

Editora Científica: Cristiane Helena Gallasch 
de 2002. A violência sexual contra as mulheres pode acarretar danos em sua saúde física, mental, sexual e reprodutiva ${ }^{2,3}$. Em estudo realizado na Tailândia, a taxa média de violência sexual por parceiro íntimo era de $25,4 \%{ }^{4}$. Isto revela que este é um problema mundial e que o Brasil precisa avançar em suas políticas para redução e controle da incidência nacional.

Com a finalidade de reduzir os danos às mulheres relacionados à violência sexual sofrida é necessário o atendimento por uma equipe multidisciplinar capacitada e acolhedora ${ }^{5}$. A Enfermagem é considerada responsável pelo cuidado nos diversos níveis de atenção à saúde, garantindo assistência ao indivíduo de forma integral e individualizada ${ }^{6}$.

Neste sentido, sendo a violência sexual um problema de saúde pública que se mantém incidente, permeando algumas décadas no Brasil, o setor saúde cada vez mais especializado e o avanço científico da enfermagem, faz-se necessário a ampliação dos estudos para o avanço científico teórico e prático sobre a temática. Diante da importância e atual necessidade de um olhar sensível dos profissionais para o atendimento às mulheres em situação de violência sexual, considera-se que a produção do conhecimento científico em enfermagem acerca desse tipo de violência subsidiará o processo de formação profissional, auxiliando na discussão da problemática.

Diante do exposto objetivou-se descrever a atuação do enfermeiro no atendimento às mulheres em situação de violência sexual a partir da literatura.

\section{MÉTODO}

Trata-se de uma revisão integrativa de literatura, que consiste em um método de pesquisa que possibilita a síntese da produção a respeito de uma área de conhecimento que está sendo estudada, realizada de maneira ordenada e sistemática, através da qual objetiva-se contribuir para o conhecimento, identificando uma possível lacuna do conhecimento. Para sua construção foram seguidas as seis etapas propostas: 1 - identificação do tema e seleção da hipótese ou questão de pesquisa; 2 - definição dos critérios para inclusão e exclusão de estudos; 3 - definição das informações a serem extraídas dos estudos selecionados; 4 - avaliação dos estudos incluídos; 5 - interpretação dos resultados; e 6 - apresentação da revisão ${ }^{7}$.

Na primeira etapa definiu-se o tema e a construção da questão norteadora da revisão: quais as evidências científicas acerca da prática assistencial do enfermeiro no atendimento às mulheres em situação de violência sexual?

Para a escolha dos descritores utilizados foram realizadas consultas ao Medical Subject Headings (MeSH) e Descritores em Ciências da Saúde (DECs) e após exaustivas buscas os descritores utilizados foram: "Nursing"; "Enfermagem"; Sex Offenses"; "Sexual Violence"; "Sexual Abuse"; "Rape"; "Violence Against Women"; "Delitos Sexuales"; "Agresión Sexual"; "Atentado contra el Pudor"; "Ofensas Sexuales"; "Crimes Sexuais"; "Delitos de Discriminação Sexual"; "Ofensa Sexual"; "Violência Sexual"; "Injúria Sexual"; "Delito Sexual"; "Violación"; "Estupro"; "Violencia contra la Mujer"; "Crímenes contra las Mujeres"; "Violência contra a Mulher"; "Crimes contra as Mulheres"; "Delitos contra a Mulher"; "Violência contra as Mulheres"; "Violência Doméstica e Sexual contra a Mulher". Combinados com os operadores booleanos AND e OR.

As bases de dados escolhidas foram Cumulative Index to Nursing and Allied Health Literature (CINAHL), Medical Literature on Line (MEDLINE) via portal PubMed, Scopus, Web Of Science, Literatura da América Latina e Caribe (LILACS), Base de Dados de Enfermagem (BDENF) via Portal Regional da Biblioteca Virtual em Saúde (BVS). O levantamento também foi realizado no portal eletrônico Scientific Eletronic Library Online (SciELO).

Na segunda etapa determinou-se os critérios de inclusão e exclusão dos estudos. Os critérios de inclusão dos artigos para análise foram: grupo populacional de mulheres, ser artigo decorrente de pesquisa original publicado entre 2015 e 2019, na íntegra, nos idiomas português, inglês ou espanhol, que versasse acerca da assistência de enfermagem às mulheres em situação de violência sexual. Foram exclusos dissertações, teses, artigos editoriais, de opinião, artigos duplicados, outras revisões e publicações que não tratassem acerca do referido tema. Esta coleta foi realizada no mês de abril de 2020.

Na terceira etapa, foram definidas as informações a serem extraídas dos estudos selecionados. As informações que foram extraídas dos estudos selecionados foram: título, objetivo do estudo; ano de publicação; procedência; delineamento do estudo (participantes); elementos da atuação do enfermeiro e Nível de Evidência (NE) conforme classificação proposta pelo sistema de classificação de evidência científica de acordo com a abordagem metodológica ${ }^{8}$. As informações extraídas foram inicialmente organizadas em planilhas e inseridas em um quadro.

Na quarta etapa avaliaram-se detalhadamente os estudos selecionados. Na quinta etapa, foi realizada a interpretação dos resultados, com a descrição dos principais resultados do estudo. A sexta etapa constituiu-se na apresentação da revisão por meio do presente artigo através do qual são descritos os principais resultados obtidos, evidenciados por meio da análise dos artigos incluídos. 


\section{RESULTADOS}

\section{Conhecendo os estudos}

Identificou-se nas referidas bases de dados um total de 204 estudos. O processo de busca na literatura seguiu as recomendações PRISMA ${ }^{9}$. Desses, foram excluídos 53 artigos duplicados, restando 151 artigos. Em seguida, observando os critérios de inclusão e exclusão, realizou-se a leitura dos títulos e resumos, e excluiu-se 136 artigos, restando 15 artigos elegíveis de acordo com os critérios de inclusão. Iniciou-se a leitura integral e em profundidade desses estudos por dois revisores de forma independente. Quando houve discordância entre os avaliadores, foram realizadas discussões entre os autores e resolvidas mediante consenso, resultando em uma amostra final de dez artigos. Este processo é ilustrado na Figura 1.

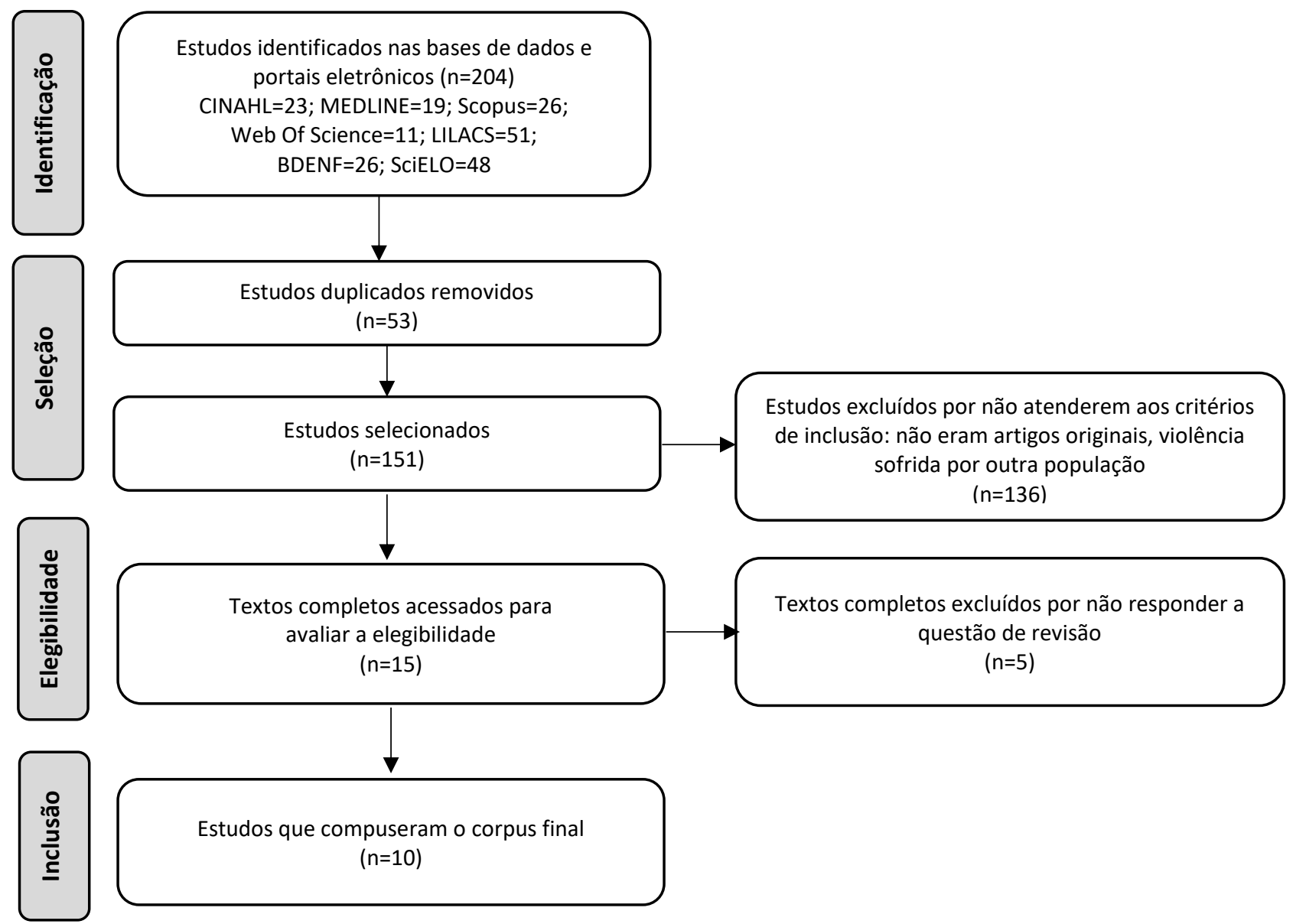

FIGURA 1: Fluxograma do processo de busca e seleção dos estudos, baseado no modelo Prisma9 ${ }^{9}$. Florianópolis, SC, Brasil, 2020.

Ao analisar o delineamento das pesquisas na amostra estudada, identificou-se que apenas um estudo foi desenvolvido com abordagem quantitativa e nove com abordagem qualitativa, com a utilização de alguns referenciais teóricos, tais como: fenomenologia, teoria holística, entre outros. Desta forma, os dados coletados serão discutidos de maneira qualitativa.

Durante a análise dos dados, a partir da avaliação da contribuição das pesquisas desenvolvidas acerca da atuação do enfermeiro no atendimento às mulheres em situação de violência sexual, observou-se moderado nível de evidências científicas dos estudos, considerando a totalidade dos artigos que possuem classificação com Nível de Evidência $V$, segundo o sistema de classificação de evidências que categoriza os estudos de forma hierárquica de acordo com a abordagem metodológica ${ }^{8}$. Isso se deve ao fato de quase todos os estudos incluídos serem de abordagem qualitativa, o que não significa que tenham menos valor, mas isso está relacionado ao fato de este tipo de estudos não ser generalizável.

\section{Síntese dos resultados}

Foi elaborado um quadro para a ordenação dos 10 artigos selecionados, contendo as seguintes variáveis: referências (autores/ano), objetivo, participantes, delineamento, síntese dos resultados encontrados e nível de evidência (Figura 2). 


\begin{tabular}{|c|c|c|c|c|c|}
\hline Referência & Objetivo & Participantes & Delineamento & Síntese dos resultados encontrados & $\begin{array}{l}\text { Nível de } \\
\text { Evidência }\end{array}$ \\
\hline $\begin{array}{l}\text { Barros et al. } \\
(2015)^{10}\end{array}$ & $\begin{array}{l}\text { Conhecer a estrutura e } \\
\text { funcionamento dos serviços } \\
\text { de saúde na perspectiva das } \\
\text { mulheres que sofreram } \\
\text { estupro. }\end{array}$ & $\begin{array}{l}11 \text { Mulheres que } \\
\text { vivenciaram a } \\
\text { violência sexual }\end{array}$ & Qualitativo & $\begin{array}{l}\text { Qualificação nas práticas do acolhimento } \\
\text { e estratégias para organização do } \\
\text { serviço, facilitando o processo de } \\
\text { enfermagem, garantindo o registro das } \\
\text { intervenções realizadas. }\end{array}$ & $\mathrm{V}$ \\
\hline $\begin{array}{l}\text { Silva, Padoin e } \\
\text { Vianna }(2015)^{11}\end{array}$ & $\begin{array}{l}\text { Conhecer e analisar as } \\
\text { concepções de profissionais } \\
\text { das equipes de saúde da } \\
\text { família em relação à } \\
\text { violência contra } \\
\text { a mulher e à prática } \\
\text { assistencial }\end{array}$ & $\begin{array}{l}30 \text { Profissionais de } \\
\text { Saúde }\end{array}$ & Qualitativo & $\begin{array}{l}\text { A prática assistencial parte de uma } \\
\text { concepção biológica do problema } \\
\text { com tendência à social. Os cuidados } \\
\text { direcionam-se ao tratamento da } \\
\text { lesão física, escuta, orientação à } \\
\text { denúncia e encaminhamento a } \\
\text { setores especializados de saúde e } \\
\text { assistência social. }\end{array}$ & V \\
\hline $\begin{array}{l}\text { Baptista et al. } \\
(2015)^{12}\end{array}$ & $\begin{array}{l}\text { Investigar a prática dos } \\
\text { enfermeiros acerca da } \\
\text { violência sexual contra } \\
\text { mulheres. }\end{array}$ & 27 Enfermeiros (as) & Quantitativo & $\begin{array}{l}\text { Os profissionais sabem do seu papel } \\
\text { de identificar a violência sexual, mas } \\
\text { em sua maioria não se sentem } \\
\text { preparados para atuarem diante de } \\
\text { uma situação como esta. }\end{array}$ & V \\
\hline $\begin{array}{l}\text { Cortes et al. } \\
(2015)^{13}\end{array}$ & $\begin{array}{l}\text { Conhecer as ações de cuidar } \\
\text { de mulheres em situação de } \\
\text { violência por enfermeiras } \\
\text { em serviços de urgência e } \\
\text { emergência e analisar as } \\
\text { ações que busquem o } \\
\text { empoderamento de } \\
\text { mulheres para a equidade } \\
\text { de gênero. }\end{array}$ & 10 Enfermeiras & Qualitativo & $\begin{array}{l}\text { Cuidado clínico relacionado a } \\
\text { administração medicamentosa, } \\
\text { conversa, escuta e orientação. }\end{array}$ & V \\
\hline $\begin{array}{l}\text { Reis, Lopes e } \\
\text { Osis }(2016)^{14}\end{array}$ & $\begin{array}{l}\text { Descrever as experiências } \\
\text { de mulheres que sofreram } \\
\text { violência sexual e o impacto } \\
\text { e importância dessa } \\
\text { violência em suas vidas. }\end{array}$ & $\begin{array}{l}11 \text { Mulheres que } \\
\text { vivenciaram a } \\
\text { violência sexual }\end{array}$ & Qualitativo & $\begin{array}{l}\text { Os enfermeiros são responsáveis pelo } \\
\text { acolhimento das mulheres na sala de } \\
\text { emergência e por todas as consultas de } \\
\text { acompanhamento. }\end{array}$ & V \\
\hline $\begin{array}{l}\text { Cortes e Padoin } \\
(2016)^{15}\end{array}$ & $\begin{array}{l}\text { Apreender as motivações da } \\
\text { ação da enfermeira ao } \\
\text { cuidar de mulheres em } \\
\text { situação de violência. }\end{array}$ & 10 enfermeiras & Qualitativo & $\begin{array}{l}\text { A busca inicial da recuperação da saúde } \\
\text { física das mulheres, proporcionando } \\
\text { bem-estar emocional, apoio e a } \\
\text { continuidade do cuidado. }\end{array}$ & V \\
\hline $\begin{array}{l}\text { Trigueiro et al. } \\
(2017)^{16}\end{array}$ & $\begin{array}{l}\text { Compreender as ações do } \\
\text { cotidiano de mulheres que } \\
\text { vivenciaram violência } \\
\text { sexual. }\end{array}$ & $\begin{array}{l}11 \text { Mulheres que } \\
\text { vivenciaram a } \\
\text { violência sexual }\end{array}$ & Qualitativo & $\begin{array}{l}\text { Evidenciou-se o sofrimento psíquico, } \\
\text { traduzido pelo medo. Para superar as } \\
\text { consequências deste tipo de violência } \\
\text { sexual, as mulheres buscam apoio de } \\
\text { familiares e amigos e a reinserção no } \\
\text { mercado de trabalho e na escola. }\end{array}$ & V \\
\hline $\begin{array}{l}\text { Fornari e } \\
\text { Labronici } \\
(2018)^{17}\end{array}$ & $\begin{array}{l}\text { Conhecer o processo de } \\
\text { resiliência em mulheres } \\
\text { vítimas de violência sexual. }\end{array}$ & $\begin{array}{l}12 \text { Mulheres que } \\
\text { vivenciaram a } \\
\text { violência sexual }\end{array}$ & Qualitativo & $\begin{array}{l}\text { O cuidado da enfermagem em sua } \\
\text { escuta nos serviços de apoio institucional } \\
\text { promoveu ajuda na recuperação das } \\
\text { mulheres. }\end{array}$ & V \\
\hline $\begin{array}{l}\text { Trentin et al. } \\
(2018)^{18}\end{array}$ & $\begin{array}{l}\text { Investigar como os } \\
\text { profissionais realizam a } \\
\text { abordagem a mulheres em } \\
\text { situação de violência sexual, } \\
\text { na perspectiva da Bioética } \\
\text { de Intervenção. }\end{array}$ & $\begin{array}{l}30 \text { profissionais, } \\
\text { dentre eles } \\
\text { advogado, } \\
\text { assistente social, } \\
\text { enfermeiro, médico, } \\
\text { psicólogo e técnicos } \\
\text { de enfermagem. }\end{array}$ & Qualitativo & $\begin{array}{l}\text { Comprometimento profissional com } \\
\text { trabalho em equipe e intersetorialidade }\end{array}$ & V \\
\hline $\begin{array}{l}\text { Netto et al. } \\
(2018)^{19}\end{array}$ & $\begin{array}{l}\text { Analisar pela ótica da Teoria } \\
\text { de Enfermagem de Levine, o } \\
\text { atendimento da enfermeira } \\
\text { às mulheres que sofreram } \\
\text { violência. }\end{array}$ & 11 Enfermeiras & Qualitativo & $\begin{array}{l}\text { Conservação de energia, integridade } \\
\text { estrutural, pessoal e social das mulheres } \\
\text { em situação de violência. }\end{array}$ & V \\
\hline
\end{tabular}

FIGURA 2: Descrição da produção científica incluída na revisão integrativa. Florianópolis, SC, Brasil, 2020. 
Com relação aos objetivos dos estudos, tiveram como foco conhecer/analisar o atendimento de enfermagem às mulheres em situação de violência sexual e violência em um contexto mais amplo. A leitura completa dos estudos permitiu observar que os objetivos propostos nos estudos originais foram alcançados (síntese dos resultados encontrados). No que diz respeito aos participantes dos estudos qualitativos, participaram 163 pessoas, das quais 58 $(35,6 \%)$ eram enfermeiras (os), 45 (27,60\%) mulheres que vivenciaram violência e 60 (36,80\%) participantes eram advogados, assistentes sociais, enfermeiros, médicos, psicólogos e técnicos de enfermagem. As participações ocorreram por meio de entrevistas semiestruturadas, questionários e grupo focal.

A análise dos dados revelou a produção do conhecimento científico existente acerca da assistência de enfermagem às mulheres em situações de vulnerabilidade, relacionadas à violência sexual. Os resultados apresentam as principais práticas realizadas na assistência a esse grupo, sendo categorizadas como a assistência clínica medicamentosa, a assistência clínica não-medicamentosa e a falta de qualificação profissional, revelando insatisfação de algumas mulheres no atendimento.

A assistência clínica medicamentosa norteada pelos protocolos, direcionam os cuidados imediatos a serem realizados com as mulheres, no propósito de evitar e/ou minimizar danos fisiológicos/sistêmicos.

A assistência clínica não-medicamentosa é desenvolvida pela equipe multiprofissional envolvida no atendimento. Isto acontece por meio de um atendimento respeitoso, sem revitimizá-las, proporcionando apoio emocional e desenvolvendo sua autoestima.

A falta de qualificação profissional, revelando insatisfação de algumas mulheres no atendimento, sendo que a necessidade da qualificação profissional é justificada pelos dados presentes nos estudos desta revisão, que mostram que as enfermeiras não recebem treinamento específico para este tipo de atendimento, e ao se depararem com tais situações em seus ambientes de trabalho sentem dificuldades para executarem os protocolos já estabelecidos.

\section{DISCUSSÃO}

A análise dos dados revelou a produção do conhecimento científico existente acerca da assistência de enfermagem às mulheres em situações de vulnerabilidade, relacionadas à violência sexual. Os resultados apresentam as principais práticas realizadas na assistência a esse grupo, sendo elas caracterizadas como a assistência clínica medicamentosa e a assistência clínica não-medicamentosa, e apontam, ainda, a falta de qualificação profissional, revelando insatisfação de algumas mulheres no atendimento.

O controle/prevenção das Infecções Sexualmente Transmissíveis (IST) é o foco inicial da assistência clínica prestada pelos profissionais de enfermagem, a fim de garantir a integridade estrutural das mulheres agredidas sexualmente, sendo o medo de desenvolver essas IST motivo para fragilizá-las ainda mais, que é acentuado pelo pensamento da dificuldade de criar um filho com o Vírus da Imunodeficiência Humana (HIV), por exemplo, diante da possibilidade de uma gestação. Além das solicitações de exames laboratoriais, o enfermeiro durante sua prática clínica poderá identificar, a partir da anamnese, lesões de pele que devem ser tratadas para evitar sua permanência ${ }^{10,13,16,19}$

A assistência de enfermagem realizada é considerada como técnica. Esta, sempre protocolada nos serviços responsáveis pelo atendimento, é pautada em condutas direcionadas às questões biológicas. O manejo é rotineiramente realizado a partir da avaliação dos padrões dos sinais vitais, quimioprofilaxia do HIV e sorologias, administração do anticoncepcional de emergência e administração de vacina e imunoglobulina contra hepatite $\mathrm{B}^{10,11,13,15}$. O cuidado das lesões de pele das mulheres estupradas também é responsabilidade e atribuição da enfermagem, contribuindo para a recuperação física. Neste cuidado está inserido a realização de curativos quando há ferida aberta decorrente do estupro ${ }^{13-15}$.

Após este atendimento clínico, a enfermagem realiza orientações/encaminhamentos a outros profissionais capacitados para a continuidade do atendimento, como médicos, psicólogos e assistentes sociais, considerados na literatura como especialistas ${ }^{13-15}$. O trabalho multiprofissional com os profissionais mencionados sugere uma eficácia no atendimento referente à escuta da mulher e redução de sobrecarga de trabalho ${ }^{14,18}$.

Surge a reflexão quanto à capacidade e habilidade da enfermagem no que tange a realizar a continuidade deste atendimento, até mesmo para minimizar a exposição destas mulheres violentadas sexualmente e que passam a ser violentadas socialmente através da sua exposição e constante necessidade de reviver o estupro a cada anamnese feita com outros profissionais especialistas. Por exemplo, quando realizada a coleta de vestígios (sêmen) no corpo da mulher pelo médico, ocasião em que o procedimento poderia ser realizado por um enfermeiro com treinamento específico para tal, como o enfermeiro forense ${ }^{20}$, uma vez que este possui caráter e habilidade para o atendimento a esse público.

Realizada a operacionalização de protocolos clínicos pelos profissionais é iniciada a escuta e diálogo com essas mulheres, na perspectiva de alcançar o bem-estar emocional delas, prevenindo possíveis agravos psicológicos 
decorrentes do estupro. Este é um momento de troca, em que as mulheres se aliviam através do desabafo e os enfermeiros conseguem realizar as devidas orientações, sejam elas clínicas ou legais, transmitindo segurança às mulheres que se encontram vulneráveis e orientando a necessidade do apoio familiar ${ }^{10,15}$.

Estudo revela que as mulheres além da execução de protocolos esperam por um acolhimento digno e respeitoso que previna a revitimização. A enfermagem assim, exerce também o papel de proporcionar apoio emocional, motivar e proporcionar a autoestima ${ }^{10,19}$.

Justifica-se também o diálogo como forma de evitar o julgamento por parte da equipe multiprofissional para com as mulheres, como também a curiosidade e o instinto ${ }^{15}$ distorcendo preceitos éticos. Este diálogo permite o sentimento de acolhimento por parte dos profissionais de enfermagem para com as mulheres que foram violentadas ${ }^{17}$. 0 diálogo entre profissionais e pacientes, como também junto aos acompanhantes permite o fortalecimento das mulheres diante da situação do estupro, despertando para as oportunidades e alternativas que essa mulher pode ter enquanto ser humano ${ }^{15}$

Em estudo realizado na Tailândia com a finalidade de avaliar a violência por parceiro íntimo, e que englobou os aspectos físicos, emocionais e violência sexual vivenciados pelas mulheres, corrobora com o fato de que o diálogo e a atenção dada pelos profissionais de enfermagem e familiares são benéficos e reduzem os sintomas físicos e depressivos. Porém, para a amostra do estudo, o efeito mediador foi caracterizado como fraco ${ }^{4}$.

Em 2015, um estudo realizado com 27 enfermeiras da atenção primária à saúde, revelou-se que rotineiramente estas, em seu atendimento, não realizavam questionamentos quanto à violência sexual, representando $77,8 \%$ da amostra do estudo, ainda que $85,1 \%$ já tenham realizado atendimentos às mulheres que vivenciaram a violência sexual. Ainda, $88,8 \%$ não utilizaram protocolo de atendimento e $62,9 \%$ fizeram encaminhamentos para outros serviços. Constatou-se que as profissionais não receberam treinamento específico para este atendimento e alegavam não possuir protocolos que favoreçam o atendimento, além de desconhecer os aspectos éticos e legais ${ }^{12}$

Outro estudo revelou a existência de protocolos de atendimentos definidos, mas também a falta de habilidade dos profissionais para executar os procedimentos, fato identificado a partir da postura profissional permeada por julgamento e culpabilização da mulher. Tais atitudes suscitaram insatisfação das pacientes ${ }^{17}$.

O comprometimento profissional deve "transcender o limite da sua função além da formação técnica profissional" e a fragilidade de conhecimento compromete a qualidade da abordagem utilizada com a vítima de violência sexual ${ }^{18}$. As mulheres atendidas por equipes sem preparo têm relatado queixas referentes à morosidade no início e continuidade do atendimento, falta de infraestrutura para o atendimento, como a passagem por outros profissionais e conversas paralelas, demonstrando falta de ética e respeito para com elas, além da insegurança dos profissionais que realizam o atendimento ${ }^{10}$

A enfermagem participa do acompanhamento das mulheres após o atendimento inicial, em consultas agendadas após o primeiro atendimento com 7, 21, 30, 45, 60, 90 e 180 dias no serviço especializado para acompanhamento

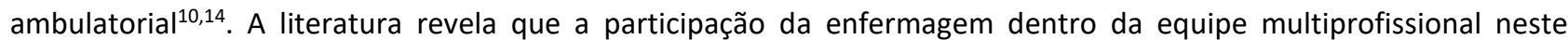
acompanhamento se dá através da organização do serviço, elaboração de protocolos para sistematização do atendimento e no registro das intervenções cabíveis e necessárias realizadas ${ }^{10}$.

Observa-se a evolução das políticas e procedimentos referentes ao atendimento às mulheres em situação de violência sexual e o papel da enfermagem nesse processo de atendimento, porém ainda é necessária uma "reestruturação organizacional e intersetorial" 21 . Como exemplo, nos Estados Unidos e Canadá, a especialidade de enfermagem Forense possui treinamento específico para atendimento em casos de agressões sexuais através do curso Sexual Assault Nurse Examiner (SANE) há quase três décadas. Nos Estados Unidos o Enfermeiro Forense examinador de agressão sexual, além de realizar as condutas já realizadas no Brasil, também é responsável pela coleta de evidências por meio da coleta de material biológico deixada pelo perpetrador, não sendo necessária a participação do profissional médico, a partir de treinamento específico para o atendimento integral das mulheres fornecido pela especialidade ${ }^{22}$.

O direito das mulheres ao aborto quando a gestação for causada pelo estupro é regulamentado em lei. Nacionalmente, o Conselho Federal de Enfermagem não obriga o profissional de sua categoria a participar do procedimento, sendo facultativo ao mesmo participar com base em seus valores e crenças. Nessa perspectiva, questiona-se onde fica a imparcialidade necessária para a profissão? Este questionamento é levantado a partir da ausência de estudos a respeito da interrupção legal da gestação relacionada à violência sexual.

\section{CONCLUSÃO}

Os estudos revisados permitiram descrever a atuação do enfermeiro no atendimento às mulheres em situação de violência sexual a partir da literatura. 
Os resultados evidenciam as principais práticas realizadas pelo enfermeiro na assistência direcionada à esta população específica, sendo categorizadas como um conjunto de ações que envolvem: assistência clínica medicamentosa, norteada por protocolos, direcionando os cuidados imediatos a serem realizados com as mulheres, com o propósito de evitar e/ou minimizar danos fisiológicos/sistêmicos; assistência clínica não-medicamentosa, desenvolvida pela equipe multiprofissional, por meio de um atendimento respeitoso, sem revitimizar as mulheres, proporcionando-Ihes apoio emocional e desenvolvendo sua autoestima.

Evidenciou-se a falta de qualificação profissional, motivo de insatisfação de algumas mulheres no atendimento, sendo que a necessidade da qualificação profissional é justificada pelos dados presentes nos estudos desta revisão, que mostram que as enfermeiras não recebem treinamento específico para este tipo de atendimento, e ao se depararem com tais situações em seus ambientes de trabalho sentem dificuldades, inclusive para executarem os protocolos já estabelecidos.

Os estudos analisados abordam com clareza a assistência de enfermagem prestada de imediato às mulheres pós agressão sexual, porém, não abordam o aprofundamento relacionado à continuidade do processo, constituindo-se essa uma importante lacuna do conhecimento. Por fim, a síntese de conhecimento produzido nesse estudo oferece subsídios valiosos para a melhoria da qualidade da assistência de enfermagem às mulheres em situação de violência e ratifica a importância dos seus resultados para fundamentar a prática clínica nesta área de estudo.

\section{REFERÊNCIAS}

1. Minayo MCS, Souza ER, Silva MMA, Assis SG. Institutionalizing the theme of violence within Brazil's national health system: progress and challenges. Ciênc. saúde coletiva [Internet]. 2018 [cited 2019 Oct 28]; 23(6):2007-16. DOI: http://dx.doi.org/10.1590/1413-81232018236.04962018.

2. World health statistics 2018: monitoring health for the SDGs, sustainable development goals. Geneva: World Health Organization; 2018.

3. Krug EG et al., eds. World report on violence and health. Geneva, World Health Organization, 2002.

4. Ross R, Stidham AW, Saenyakul P, Creswell JW. Intimate partner violence, emotional support and health outcomes among Thai women: a mixed methods study. Journal Of The Royal Thai Army Nurses [Internet]. 2015 [cited 2019 Oct 28]; 16(1):22-32. Available from:

https://www.researchgate.net/publication/276265431_Intimate_partner_violence_emotional_support_and_health_outcomes _among_Thai_women_A_mixed_methods_study.

5. Ministério da Saúde (BR). Portaria Ministerial no 485, de 1o de abril de 2014. Redefine o funcionamento do Serviço de Atenção às Pessoas em Situação de Violência Sexual no âmbito do Sistema Único de Saúde (SUS). Diário Oficial [da República Federativa do Brasil], Brasília, DF, 02 abr. 2014. Seção I, no 63. p 53. Available from: http://bvsms.saude.gov.br/bvs/saudelegis/gm/2014/prt0485_01_04_2014.html.

6. Moser DC. Sistematização da assistência de enfermagem: percepção dos enfermeiros. J. res.: fundam. care. Online. 2018 [cited 2019 Oct 28]; 10(4):998-1007. DOI: https://doi.org/10.9789/2175-5361.2018.v10i4.998-1007.

7. Mendes KDS, Silveira RCCP, Galvão CM. Integrative literature review: a research method to incorporate evidence in health care and nursing. Texto contexto - enferm. 2008 [cited 2021 May 28]; 17(4):758-64. DOI: https://doi.org/10.1590/S010407072008000400018.

8. Melnyk BM, Fineout-Overholt E. Making the case for evidences-based practice. In: Melnyk, BM, editor. Fineout-Overholt E. Making the case for evidences-based practice in nursing \& healthcare: a guide to best practice. Philadelphia: Lippincot Willians \& Willkins; 2005. Pp. 3-24

9. Moher D, Liberati A, Tetzlaff J, Altman DG; PRISMA Group. Preferred reporting items for; systematic reviews and meta-analyses: the PRISMA statement. PloS Med. 2009; 6(7):e1000097. DOI: https://doi.org/10.1371/journal.pmed.1000097.

10. Barros LA, Albuquerque MCS, Gomes NP, Riscado JLS, Araújo BRO, Magalhães JRF. The (un)receptive experiences of female rape victims who seek healthcare services. Rev. esc. enferm. USP [Internet]. 2015 [cited 2019 Oct 28]; 49(2):193-200. DOI: http://dx.doi.org/10.1590/S0080-623420150000200002.

11. Silva EB, Padoin SMM, Vianna LAC. Violence against women and care practice in the perception of the health professionals. Texto contexto - enferm. [Internet]. 2015 [cited 2019 Oct 28]; 24(1):229-37. DOI: http://dx.doi.org/10.1590/010407072015003350013.

12. Baptista RS, Chaves OBBM, França ISX, Sousa FS, Oliveira MG, Leite CCS. Sexual violence against women: nurses' practice. Rev. Rene [Internet]. 2015 [cited 2019 Oct 28]; 16(2):210-7. DOI: http://dx.doi.org/10.15253/2175-6783.2015000200010.

13. Cortes LF, Padoin SMM, Vieira LB, LanderdahI MC, Arboit J. Care for women victims of violence: empowering nurses in the pursuit of gender equity. Rev. Gaúcha Enferm. [Internet]. 2015 [cited 2019 Oct 28]; 36(spe): 77-84. DOI: http://dx.doi.org/10.1590/1983-1447.2015.esp.57162.

14. Reis MJ, Lopes MHBM, Osis MJD. 'It's much worse than dying': the experiences of female victims of sexual violence. Journal Of Clinical Nursing [Internet]. 2016 [cited 2019 Oct 28]; 26(15-16):2353-61. DOI: http://dx.doi.org/10.1111/jocn.13247.

15. Cortes LF, Padoin SMM. Intentionality of the action of caring for women in situations of violence: contributions to nursing and health. Esc. Anna Nery. 2016 [cited 2019 Oct 28]; 20(4):e20160083. Available from: https://www.scielo.br/j/ean/a/b8Yz6Yvh5tskjfFrnrgWnWv/?lang=en 
16. Trigueiro TH, Silva MH, Merighi MAB, Oliveira DM, Jesus MCP. Psychological suffering in the daily lives of women who have experienced sexual violence: a phenomenological study. Esc Anna Nery 2017 [cited 2019 Oct 28]; 21(3):e20160282. Available from: https://www.scielo.br/j/ean/a/T5t5FvWMDfx6tNfDK97g3pH/?lang=en.

17. Fornari LF, Labronici LM. The process of resilience in women who were victims of sexual violence: a possibility for care. Cogitare Enferm. 2018 [cited 2019 Oct 28]; 23(1:):e52081. DOI: http://dx.doi.org/10.5380/ce.v23i1.52081.

18. Trentin D, Oliveira VMA, Pires de Pires DE, Hellmann F, Brehmer L, Cézar Leal S. Attention to women in the situation of sexual violence in the bioethics perspective. Acta bioeth. [Internet]. 2018 [cited 2019 Oct 28]; 24(1):117-26. DOI: http://dx.doi.org/10.4067/S1726-569X2018000100117.

19. Netto LA, Pereira ER, Tavares JMAB, Ferreira DC, Broca PV. Nursing performance in the conservation of women's health in situations of violence. REME - Rev. Min. Enferm. 2018 [cited 2019 Oct 28]; 22:e-1149. DOI: http://dx.doi.org/10.5935/14152762.20180080 .

20. COFEN. Resolução no 556, de 23 de agosto de 2017. Regulamenta a atividade do Enfermeiro Forense no Brasil, e dá outras providências. Resolução Cofen № 566/2017. Brasília, DF. Available from: http://www.cofen.gov.br/resolucao-cofen-no05562017_54582.html.

21. Mafioletti TM, Peres AM, Larocca LM, Fontoura MP. Violence against women: historical trajectory of a care program (Curitiba 1997-2014). Rev. Bras. Enferm. [Internet]. 2018 [cited 2019 Oct 28]; 71(6): 2907-15. DOI: http://dx.doi.org/10.1590/00347167-2017-0583.

22. Campbell R, Bybee D, Kelley KD, Dworkin ER, Patterson D. The impact of sexual assault nurse examiner (SANE) Program Services on Law Enforcement Investigational Practices. Criminal Justice And Behavior [Internet]. [cited 2019 Oct 28]; 39(2):169-184. DOI: http://dx.doi.org/10.1177/0093854811428038. 